\section{Estudo \\ CoDebate}

em Testão

Planejamento
Revista Estudo \& Debate, Lajeado, v. 25, n. 1, 2018. ISSN 1983-036X

DOI: http://dx.doi.org/10.22410/issn.1983-036X.v25i1a2018.1655

\title{
ÍNDICE DE DESENVOLVIMENTO ECONÔMICO DAS FAMÍLIAS NO RIO GRANDE DO SUL: UMA ABORDAGEM POR INTERMÉDIO DA ANÁLISE MULTIVARIADA
}

\author{
Raquel Pereira Pontes ${ }^{1}$, João Eustáquio de Lima²
}

\begin{abstract}
Resumo: Este estudo tem como objetivo analisar o Índice de Desenvolvimento Econômico das Famílias (IDF) do Estado do Rio Grande do Sul por meio da Análise Multivariada. O estudo também busca obter esse índice pelos escores fatoriais das dimensóes latentes encontradas pela Análise Fatorial Exploratória além de, oportunamente, analisar estas famílias por grupo através do método de agrupamento. $\mathrm{O}$ interesse neste índice é poder observar o perfil socioeconômico dos domićlíos do Rio Grande do Sul, preocupaçáo justificada devido à crise financeira pela qual atravessa o referido ente federativo nos últimos anos. Os resultados apresentaram

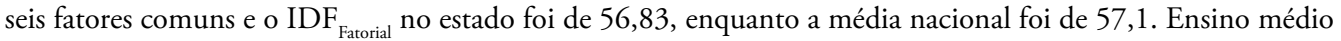
completo e estar empregado no setor formal são características de grupos com maior IDF. Famílias que residem em área rural ou com presença de idosos apresentaram menor IDF. Indicadores demonstraram que apesar de $48,8 \%$ dos domicílios terem pelo menos um ocupado no setor formal, $98 \%$ das famílias estáo acima da linha da pobreza e $69 \%$ têm capacidade de geração de renda. Indicadores como ausência de mortalidade infantil e progresso escolar também se destacaram nos resultados.
\end{abstract}

Palavras-chave: Desenvolvimento Econômico; Índice; Análise Multivariada.

\section{INDEX OF ECONOMIC DEVELOPMENT OF FAMILIES IN RIO GRANDE DO SUL: AN APPROACH THROUGH MULTIVARIATE ANALYSIS}

\begin{abstract}
This study aims to analyze the Index of Economic Development of Families (IDF) of the State of Rio Grande do Sul through the Multivariate Analysis. The study also seeks to obtain this index by factorial scores of latent dimensions found by the Exploratory Factor Analysis and, in a timely manner, to analyze these families by group using the grouping method. The interest in this index is to be able to observe the socioeconomic profile of the households in Rio Grande do Sul, a justified concern due the financial crisis through which the federative entity is facing in recent years. The results presented six common factors and the $\operatorname{IDF}_{\text {Factorial }}$ in the state was 56.83, while the national average was 57.1. High school and formal education are characteristics of
\end{abstract}

1 Doutoranda em Economia Aplicada - Departamento de Economia Rural - UFV. Mestre em Economia Aplicada - FURG. MBA em Gestão do Agronegócio - UFPR e Graduada em Ciências Econômicas - FURG.

2 Professor titular do Departamento de Economia Rural da Universidade Federal de Viçosa. Doutor em Economia Rural - Michigan State University. 
groups with higher IDF. Households living in rural areas or with the presence of the elderly showed lower IDF. Indicators showed that although $48.8 \%$ of households have at least one employed in the formal sector, $98 \%$ of households are above the poverty line and $69 \%$ have income generation capacity. Indicators such as absence of infant mortality and school progress also stood out in the results.

Keywords: Economic Development; Index; Multivariate analysis.

\section{INTRODUÇÁO}

Atualmente, há discussão na literatura sobre como analisar e identificar a situação de vulnerabilidade socioeconômica de determinados segmentos populacionais. Alguns autores como Rocha (1997) e Falcão e Costa (2014) sugerem uma análise unidimensional, na qual a renda é o principal fator para a caracterização da pobreza. Todavia, outros investigadores - como Chakravarty, Mukherjee e Ranade (1998), Bourguignon e Chakrvart (2003), Hoffmann e Kageyama (2006) e Silva et al. (2014) - optam por uma análise multidimensional que exige elementos além da renda para a averiguação da situaçáo de pobreza.

Ao longo do tempo foram desenvolvidos diversos indicadores sociais na tentativa de captar informaçóes sobre determinada realidade social dos indivíduos estudados. Os indicadores também almejam demonstrar às autoridades políticas quais populaçóes e/ou regiōes demandam atenção de políticas públicas e sociais, sendo o Índice de Desenvolvimento Humano (IDH) - criado na década de 1990 para comparar o desenvolvimento entre diferentes países - o mais conhecido entre os indicadores (OLIVEIRA, 2013). No entanto, mesmo o IDH foi alvo de críticas por apresentar somente quatro dimensóes, de modo que outros índices foram criados no intuito de compreender melhor a situação da população. No Brasil, por exemplo, em 1997 foi criado o índice de qualidade de vida por Almeida (1997) e, em 1998, foi elaborado por Cide (1998) o índice de qualidade dos municípios.

Desse modo, o presente estudo visa elaborar um Índice de Desenvolvimento Econômico da Família (IDF) para o Estado do Rio Grande do Sul com base no trabalho de Barros, Carvalho e Franco (2003). Os autores desenvolveram um índice sintético em nível de família da mesma forma que o Índice de Desenvolvimento Humano, utilizado um sistema neutro de pesos, pelo qual se obtém um indicador global através da média dos indicadores sintéticos das dimensóes. Estes, por sua vez, são a média dos indicadores sintéticos dos componentes, oriundos da média dos indicadores usados para cada componente.

Desta maneira, este estudo tem como base a metodologia de Barros, Carvalho e Franco (2003), mas com uma proposta diferente, utilizando a Análise Multivariada com $\mathrm{o}$ intuito de descobrir quais são as dimensóes latentes ${ }^{3}$ que melhor retrata a variância dos dados em determinada regiáo. Para alcançar tal objetivo, emprega-se o método de Análise Fatorial Exploratória para obter os fatores comuns e os escores fatoriais ${ }^{4}$ destes, resultando

3 No estudo de Análise Multivariada as dimensões latentes ou os fatores são definidos como uma combinação linear das variáveis utilizadas. (HAIR et al., 2009).

4 Os escores fatoriais na Análise Multivariada consistem em uma medida para cada observação de cada fator obtido (HAIR et al., 2009). 
no Índice de Desenvolvimento Econômico das Famílias 5 . De forma oportuna, também será realizada a Análise de Cluster para obter os grupos de famílias, bem como apresentar resultados recentes.

Este estudo possui os seguintes objetivos específicos: 1) identificar os perfis de condiçóes de vida dos domicílios da amostra verificando a coerência com as dimensóes do estudo de Barros, Carvalho e Franco (2003); 2) calcular o $\operatorname{IDF}_{\text {Barros }}$; 3) elaborar o Índice de Desenvolvimento das Famílias com os escores fatoriais $\left(\mathrm{IDF}_{\text {Fatorial }}\right)$ ponderados pelas variâncias dos fatores; 4) verificar a coerência do $\operatorname{IDF}_{\text {Fatorial }}$ com o IDF $\operatorname{IBarros}_{\text {e }}$ e) realizar uma análise de clusters a partir dos escores fatoriais no sentido de identificar grupos homogêneos de domicílios com condiçóes de vida semelhantes.

A análise deste estudo se concentra no Estado do Rio Grande do Sul devido à crise econômica pela qual atravessa a referida federação, ensejando uma maior situação de vulnerabilidade para as famílias gaúchas (MOREIRA, 2014). Ressalta-se que no respectivo Estado a Fundação de Economia e Estatística Siegfried Emanuel Heuser (FEE) possui um Índice de Desenvolvimento Socioeconômico (Idese) das cidades integrantes dos 28 Conselhos Regionais de Desenvolvimento ${ }^{6}$ (COREDEs). Por conseguinte, de cada COREDE (ver FEE, 2017), este se dá de forma mais agregada do que um índice próprio para o desenvolvimento familiar no Estado. A análise regional, com estudos de problemas localizados, pode ser compreendida dentro da Economia Regional, com a importância de obter o conhecimento das especificidades das regiōes (DE SOUZA, 1981; GUIMARÃES, 1997).

É apresentada a seguir uma breve discussão sobre o desenvolvimento econômico das famílias, bem como a metodologia na qual são demonstrados os dados e explanados os métodos que foram utilizados no trabalho. Na seção seguinte, são apresentados e discutidos os resultados obtidos e, por fim, a conclusão e as referências.

\section{REFERENCIAL TEÓRICO}

\subsection{Desenvolvimento Econômico das Famílias}

O desenvolvimento econômico das famílias é relevante à economia em geral. Pearce (2003) enfatiza que a economia das famílias pertence ao "terceiro sistema", denominada economia social, o qual também inclui o setor de voluntariado e uma série de associaçóes direcionadas a autoajuda, reciprocidade e propósito social. Westlund (2003) também argumenta que a Economia da Família e a Economia Social possuem o mesmo princípio

5 Para facilitar a leitura, ao longo do texto é empregado a sigla IDF referente ao Índice de Desenvolvimento das Famílias. A expressáo "Econômico" é utilizada por tratar-se de analisar o perfil socioeconômico dos domicílios.

6 Através da Lei 10.283 de 17/10/94, foi criado 28 Conselhos Regionais de desenvolvimento, para as 28 regiốes, com intuito de averiguar a situação econômica das mesmas. 
de reciprocidade, apesar da Economia Social se basear em relacionamentos abertos, não necessariamente parentais.

A família pode ser considerada como uma unidade de reprodução econômica, tendo em vista que os pais sustentam os filhos e os idosos, por consequência, futuramente os progenitores serão sustentados (DOWBOR, 2015). As condições financeiras da família, assim como o ambiente social, educacional e fraternal podem influenciar o desenvolvimento econômico de cada família, gerando um efeito spillover na economia nacional e internacional. Consequentemente, futuros resultados econômicos dependerão do andamento dos resultados de desenvolvimento econômico das famílias.

Becker (1965) identificou a família como unidade de tomada de decisão, diferenciando da maximização de utilidade por indivíduo. Posteriormente, Becker e Tomes (1986) avaliaram que os ganhos de capital humano transmitidos pelas famílias, são aniquilados em três geraçóes. Recentemente Dowber (2015) alertou sobre a mudança da estrutura familiar, na qual observou a desarticulação da família e a diminuição dos membros que compõe a mesma. $\mathrm{O}$ autor também verificou uma transformaçáo comportamental, por exemplo, que os filhos náo seguem a carreira dos pais.

Conforme analisado por Conger et al. (1992), as dificuldades financeiras afetam as emoçóes e os comportamentos dos pais, estando correlacionado a conflitos conjugais comprometendo a criaçáo e o desenvolvimento de filhos adolescentes. Por sua vez, Ferreira e Marturano (2002) observaram que crianças com problemas comportamentais conviviam em ambiente familiar com escassez de recursos.

As condiçōes econômicas do Estado induzem o bem-estar e as condiçóes socioeconômicas das famílias, seja pela oferta de vagas de emprego, seja pelas condiçóes de acesso à educação e cuidados com a saúde. Em momentos de crises financeiras, os efeitos negativos afetam o desenvolvimento econômico das famílias, podendo gerar efeitos permanentes na economia.

O referido referencial teórico incorporou conceitos da Economia da Família, de forma breve, a fim de situar o leitor da relevância da família como uma unidade de reprodução econômica.

\section{METODOLOGIA}

Nesta seção, estão descritos os procedimentos metodológicos empregados nesta pesquisa. Inicialmente realiza-se uma descrição sobre o método. Em seguida, tecese explicações sobre a Análise Fatorial e, posteriormente, discorre-se sobre o método de agrupamento (Cluster). Por fim, realiza-se esclarecimentos sobre as variáveis consideradas na análise. 


\subsection{Método}

Este estudo parte de uma Análise Estatística Multivariada ${ }^{7}$ que tem por objetivo principal reduzir a massa de dados analisados usando um número reduzido de informação que tenha representatividade da variabilidade dos dados da pesquisa. A ideia é obter dimensóes latentes que correspondam a um perfil relevante dos dados, utilizando o método de Análise Fatorial Exploratória com a técnica de componentes principais à estimação das cargas fatoriais. A partir desse método, estimou-se os escores fatoriais, os quais foram utilizados para a construção do Índice de Desenvolvimento das Famílias. A fim de explorar mais os resultados, os escores fatoriais também foram empregados para a construção de grupos de domicílios conforme sua similaridade, aplicando-se o método de Análise de Agrupamento (Cluster) não hierárquico. Os métodos e o modelo são explanados nas duas próximas seçóes.

\subsubsection{Análise Fatorial}

O método de Análise Fatorial é uma técnica de Análise Multivariada e tem como objetivo representar dados de uma pesquisa por um número reduzido de variáveis, podendo ser denotadas como fatores comuns, como dimensóes, ou como variáveis latentes.

A Análise Fatorial pode ser Exploratória ou Confirmatória. Na Exploratória o objetivo é encontrar dimensóes que explicam os dados, permitindo que todas as variáveis possam se relacionar com todos os fatores (LIMA, 2017). Na Confirmatória, o pesquisador precisa de um modelo teórico no qual pretende confirmar se as dimensóes latentes realmente correspondem bem às informaçôes dos seus indicadores (LIMA, 2017). Neste estudo, a análise poderia ter sido realizada pela Confirmatória, já que existe uma representação teórica, com base no estudo de Barros, Carvalho e Franco (2003), no entanto preferiu-se utilizar o procedimento "Exploratório" a fim de descobrir quais dimensôes estariam representando a amostra. Por essa razão, é possível comparar se pela Análise Fatorial Exploratória chega-se a dimensóes parecidas com as do modelo teórico.

É importante utilizar variáveis padronizadas, pois a diferença na unidade de medida pode impactar na variabilidade dos dados. Por isso, geralmente, parte-se de uma matriz de correlação tendo o seguinte modelo (EQUAÇÃO 1) conforme Mingoti (2013, p.113):

$$
\begin{aligned}
& Z_{1}=l_{11} F_{1}+l_{12} F_{2}+\cdots+l_{1 m} F_{m}+\varepsilon_{1} \\
& Z_{2}=l_{21} F_{1}+l_{22} F_{2}+\cdots+l_{2 m} F_{m}+\varepsilon_{2} \\
& \cdots \\
& Z_{p}=l_{p 1} F_{1}+l_{p 2} F_{2}+\cdots+l_{p m} F_{m}+\varepsilon_{p}
\end{aligned}
$$

7 A Análise Estatística Multivariada consiste em vários métodos para analisar as múltiplas variáveis simultaneamente para um único relacionamento ou para um conjunto de relacionamento. $\mathrm{O}$ uso desta análise ocorre geralmente para a investigação e para a interpretação de grandes conjuntos de dados. (HAIR et al., 2009; LIMA, 2017). 
Em termos matriciais (EQUAÇÃO 2),

$$
(X-\mu)=L F+\varepsilon
$$

Onde $Z_{p x 1} \equiv(X-\mu)$, sendo $Z$ as variáveis padronizadas, $(X-\mu)$ é o vetor $p \times 1$ de elementos $X_{i}-\mu_{i}$, em que, $i=1,2, \ldots, p, X$ é o vetor de variáveis originais e $\mu$ é o vetor de médias $L$ é a matriz $p \times m$ de cargas fatoriais (correlação entre variáveis originais e fatores) e $\mathrm{F}$ é o vetor $m \times 1$ de fatores comuns (fatores que influenciam duas ou mais variáveis) e $\varepsilon$ é o erro (MINGOTI, 2013). Conforme Lima (2017, p. 41), neste modelo a variação total de uma variável pode ser explicada pelo fator comum mais fator específico (influencia somente uma única variável) e mais o erro, ou seja, variação total é igual à comunalidade (fator comum) mais a unicidade (fator específico e o erro).

O modelo da Análise Fatorial Exploratória é ortogonal e segue algumas pressuposiçóes (MINGOTI, 2013, p. 102-103): $E\left(F_{j}\right)=0$, ou seja, todos os fatores têm média zero; $\operatorname{Var}\left(F_{m \times 1}\right)=I$, os fatores não são correlacionados e têm variância igual a $01 ; E\left(\varepsilon_{p x 1}\right)=0$, os erros têm médias iguais a zero; $\operatorname{Var}\left(\varepsilon_{p x p}\right)=\Psi_{\text {pxp }}$, ou seja, os erros não são correlacionados entre si e tem variâncias diferentes e $E\left(\varepsilon F^{\prime}\right)=0$, isto é, os vetores do erro e dos fatores não são correlacionados.

Existem três técnicas mais utilizadas para a estimação da matriz das cargas fatoriais: $1^{\text {a) }}$ Método de Componentes Principais; 2a) Método do Fator Principal e; 3a) Método de Máxima Verossimilhança. A primeira delas foi adotada neste estudo, dado a busca por capturar maior parte da variância, ou seja, valores das cargas fatoriais que tenham valores de comunalidade mais próximo do valor da variância total observada (TRYFOS, 1997).

No modelo de Análise Fatorial Exploratória pode ser usado à rotação de fatores que consiste na rotação dos eixos coordenados, modificando as cargas fatoriais para facilitar a interpretação destas e também para a designação da dimensão latente. Todavia, verifica-se que podem ser usadas rotação oblíqua e rotação ortogonal, nesta pesquisa emprega-se a rotação ortogonal pela técnica varimax, por se tratar de uma forma mais simples de rotação, na qual os eixos são mantidos em 90 graus (HAIR et al., 2009). Em seguida, são estimados os escores fatoriais que equivalem ao valor de cada fator para cada observação da amostra.

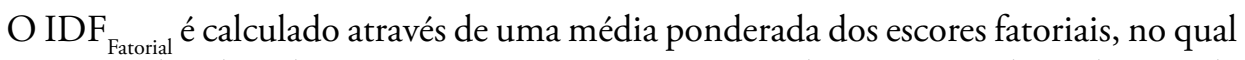
cada fator é ponderado pelas raízes características (ou seja, pela proporção de explicação da variância total associada a cada fator - peso de sua importância na variação total), de modo que (EQUAÇÃO 3):

$$
I D F_{\text {Fatorial }_{i}}=\frac{\sum_{j=1}^{r} \lambda_{j} F_{i j}}{\sum_{j=1}^{r} \lambda_{j}}, \quad i=1,2, \ldots, n \text { e } j=1,2, \ldots, r
$$

Onde, $I D F_{\text {Fatorial }}$ é o Índice de Desenvolvimento das Famílias para a família $i, \lambda_{j}$ é a raiz característica relacionada com o fator $j$ e $F_{i j}$ é o valor do escore fatorial j para a família $i, n$ é o número de observaçóes e $r$ o número de fatores. Como os escores fatoriais 
podem ter valores positivos ou negativos é necessário fazer uma transformação para que eles estejam todos no primeiro quadrante (EQUAÇÃO 4):

$$
F_{i j}^{*}=\frac{F_{i j}-F_{j \min }}{F_{j \max }-F_{j \min }}
$$

Verifica-se que $F_{i j}^{*}$ é o escore fatorial transformado no fator $j$ na família $i, F_{i j}$ é o escore fatorial do fator $j$ na família $i, F_{j m i n}$ é o menor valor do j-ésimo escore fatorial e $F_{\text {jmax }}$ é o maior valor do j-ésimo escore fatorial.

\subsubsection{Método de agrupamento (Cluster)}

O método de Cluster consiste em dividir os domicílios da amostra em diferentes grupos que possuem características semelhantes. Todavia, é preciso decidir os critérios de formação desses grupos, sendo que um deles é o próprio método de agrupamento, podendo ser hierárquico ou não hierárquico. Este estudo utilizou o método não hierárquico por ser o indicado para uma quantidade elevada de observaçóes. Entretanto, conforme sugestão de Mingoti (2013), primeiro será realizado o método hierárquico para se obter o número provável de grupos, e com a informação obtida aplicar o método não hierárquico.

A realização do método hierárquico se deu com a utilização da técnica de agrupamento pelo método de Ward para formar grupos com maior homogeneidade interna e a medida de similaridade de Distância Euclidiana. Devido ao uso dos escores fatoriais para esta estimação, observa-se que o método de Distância Euclidiana tem importantes propriedades, por exemplo, base geométrica bem definida e invariante com relação à transformação ortogonal (LIMA, 2017).

Existem técnicas dentro do método não hierárquico para gerar os grupos, na qual as mais usadas são k-médias e k-medianas. Neste estudo utilizou-se o método k-médias, pois este procedimento aloca os elementos que são próximos ao vetor de médias amostral (centroide) em cada grupo (HARTINGAN; WONG, 1979 apud MINGOTI, 2013, p. 192; LIMA, 2017). No método não hierárquico também empregou-se a Distância Euclidiana para medida de similaridade.

\subsection{Variáveis}

As variáveis consideradas para esta análise são dicotômicas, ou seja, com reposta 0 ou 1. Foram criados 48 indicadores com base na metodologia de Barros, Carvalho e Franco (2003) utilizando dados da Pesquisa Nacional de Domicílios (PNAD) do ano de 2015, realizada e disponibilizada pelo Instituto Brasileiro de Geografia e Estatística (IBGE), conforme apresentado no Quadro 1. 


\section{Quadro 1 - Indicadores do Índice de Desenvolvimento Econômico das Famílias}

\begin{tabular}{|c|c|}
\hline Dimensóes & Indicadores \\
\hline \multirow{10}{*}{$\begin{array}{c}\text { Ausência de } \\
\text { Vulnerabilidade }\end{array}$} & V1. Nenhuma mulher teve filho nascido vivo no último ano \\
\hline & V2. Nenhuma mulher teve filho nascido vivo nos últimos dois anos \\
\hline & V3. Ausência de criança \\
\hline & V4. Ausência de crianças e adolescentes \\
\hline & V5. Ausência de criança, adolescente ou jovem adulto \\
\hline & V6. Ausência de idoso \\
\hline & V7. Presença de cônjuge \\
\hline & V8. Mais da metade dos membros encontra-se em idade ativa \\
\hline & V9. Não existe criança no domicílio cuja mãe tenha morrido \\
\hline & V10. Não existe criança no domicílio que não viva com a mãe \\
\hline \multirow{6}{*}{$\begin{array}{c}\text { Acesso ao } \\
\text { Conhecimento }\end{array}$} & C1. Ausência de adulto analfabeto \\
\hline & C2. Ausência de adulto analfabeto funcional \\
\hline & C3. Presença de pelo menos um adulto com fundamental completo \\
\hline & C4. Presença de pelo menos um adulto com ensino médio completo \\
\hline & C5. Presença de pelo menos um adulto com alguma educaçáo superior \\
\hline & C6. Presença de pelo menos um trabalhador com qualificação média ou alta \\
\hline \multirow{6}{*}{$\begin{array}{l}\text { Acesso ao } \\
\text { Trabalho }\end{array}$} & T1. Mais da metade dos membros em idade ativa encontra-se ocupada \\
\hline & T2. Presença de pelo menos um trabalhador há mais de seis meses no trabalho atual \\
\hline & T3. Presença de pelo menos um ocupado no setor formal \\
\hline & T4. Presença de pelo menos um ocupado em atividade não-agrícola \\
\hline & T5. Presença de pelo menos um ocupado com rendimento superior a 1 salário mínimo \\
\hline & T6. Presença de pelo menos um ocupado com rendimento superior a 2 salários mínimos \\
\hline \multirow{3}{*}{$\begin{array}{l}\text { Disponibilidade } \\
\text { de Recursos }\end{array}$} & R1. Renda familiar per capita superior à linha de extrema pobreza \\
\hline & R2. Renda familiar per capita superior à linha de pobreza \\
\hline & R3. Maior parte da renda familiar não advém de transferências \\
\hline \multirow{11}{*}{$\begin{array}{l}\text { Desenvolvimento } \\
\text { Infantil }\end{array}$} & D1. Ausência de criança com menos de 14 anos trabalhando \\
\hline & D2. Ausência de criança com menos de 16 anos trabalhando \\
\hline & D3. Ausência de criança até 6 anos fora da escola \\
\hline & D4. Ausência de Acesso a escola criança de 7-14 anos fora da escola \\
\hline & D5. Ausência de criança de 7-17 anos fora da escola \\
\hline & D6. Ausência de criança de até 14 anos com mais de 2 anos de atraso \\
\hline & D7. Ausência de adolescente de 10 a 14 anos analfabeto \\
\hline & D8. Ausência de jovem de 15 a 17 anos analfabeto \\
\hline & D9. Ausência de mãe cujo filho tenha morrido \\
\hline & D10. Há, no máximo, uma mãe cujo filho tenha morrido \\
\hline & D11. Ausência de máe com filho nascido morto \\
\hline
\end{tabular}




\begin{tabular}{|l|l|}
\hline Dimensóes & \\
\hline \multirow{4}{*}{$\begin{array}{c}\text { Condiçóes } \\
\text { Habitacionais }\end{array}$} & H1. Domicílio próprio \\
\cline { 2 - 3 } & H2. Domicílio próprio ou cedido \\
\cline { 2 - 3 } & H6. Densidade de até 2 moradores por dormitório \\
\cline { 2 - 3 } & H4. Material de construção permanente \\
\cline { 2 - 3 } & H8. Acesso a é coletetado \\
\cline { 2 - 3 } & H9. Acesso a fogão e geladeira \\
\cline { 2 - 3 } & H10. Acesso a fogáo, geladeira, televisão ou rádio \\
\cline { 2 - 3 } & H11. Acesso a fogão, geladeira, televisão ou rádio e telefone \\
\cline { 2 - 3 } & H12. Acesso a fogão, geladeira, televisão ou rádio, telefone e computador \\
\hline
\end{tabular}

Fonte: Adaptado de Barros, Carvalho e Franco (2003).

Considerou-se como criança pessoa com até 12 anos de idade, adolescente de 13 a 17 anos de idade, adultos de 18 a 64 anos de idade, jovens adultos de 18 a 29 anos de idade e idosos comum idade de 65 anos ou mais ${ }^{9}$. A conceituação para analfabeto funcional foi descrita como indivíduo com mais de 15 anos de idade com escolaridade inferior a quatro anos. $\mathrm{O}$ trabalhador com qualificação média ou alta foi empregado ao indivíduo que estudou mais de oito anos letivo ${ }^{10}$. Os indivíduos com idade ativa foram considerados pessoas de 10 anos a 65 anos de idade.

Considerando-se o salário mínimo do ano de 2015 (ano da base de dados) de R \$ 788,00 . O limite para a extrema pobreza e para a pobreza também foi usado com base do ano de 2015, tendo como referência ao decreto no 8.232 de 31 de maio de 2014 . O referido decreto considera-se extrema pobreza a pessoa com renda familiar per capita mensal de até $\mathrm{R} \$ 77,00$ e a pobreza a pessoa com renda familiar per capita mensal de até $\mathrm{R} \$ 154,00$.

8 O intervalo de idade de 18 a 64 anos refere-se à classificaçáo de adultos, mas o indicador V5 concerne somente a jovens adultos, estando estes classificados no intervalo de 18 a 29 anos de idade.

9 Os critérios para a classificação dos intervalos de idade foram elaborados pelos autores com base no Estatuto da Criança e do Adolescente (BRASIL, 1990) e do Estatuto do Idoso (BRASIL, 2003). A idade de referência para idoso foi considerada 65 anos devido ao aumento da expectativa de vida do brasileiro. Conforme IBGE (2016) a esperança de vida para o ano de 2015 foi de 75,5 a nível nacional e de 77,5 para o estado do Rio Grande do Sul.

10 O número de anos letivos para ter uma qualificação média ou alta foi determinado oito anos devido a educação básica obrigatória no Brasil definido pela lei no 9394 de 20 de dezembro de 1996 que estabeleceu o ensino fundamental obrigatório e gratuito. E a lei no 11.274 de 6 de fevereiro de 2006 alterou para nove anos de ensino fundamental obrigatório. Desta maneira, determinou-se que o indivíduo que tivesse acima de oito anos de estudo, possuiria uma qualificação maior que a básica, podendo ser média ou alta. Foi escolhido oito anos, devido as escolas náo terem chegado ao nono ano em 2015. 


\section{RESULTADOS E ANÁLISES}

Nesta seção apresenta-se as estimaçóes e as análises, estando dividida em quatro partes. Primeiramente, apresenta-se uma estatística descritiva dos indicadores da região e posteriormente é calculado o índice pela média simples, ou seja, o $\mathrm{IDF}_{\text {Barros }}$. Depois, destacase a análise fatorial, no qual apresentando as dimensóes latentes. Em seguida, fala-se do índice obtido pela análise fatorial e por fim, trata-se da análise de cluster.

\section{1 Índice de Desenvolvimento da Família - Barros $\left(\right.$ IDF $\left._{\text {Barros }}\right)$}

Os 48 indicadores propostos para a investigação, são evidenciados na Tabela 1, através de estatísticas descritivas. Os indicadores são associados a 25 componentes e estes a seis dimensóes.

Tabela 1 - Representação dos indicadores do IDF no estado do Rio Grande do Sul para o ano de 2015

\begin{tabular}{|c|c|c|c|c|c|}
\hline Dimensáo & Componente & Indicadores & $\begin{array}{c}\text { Obs. } \\
\text { (Domicílios) }\end{array}$ & $\begin{array}{c}\text { Média } \\
(\%)\end{array}$ & $\begin{array}{l}\text { Desvio } \\
\text { Padráo }\end{array}$ \\
\hline \multirow{14}{*}{$\begin{array}{l}\text { 1. Ausência de } \\
\text { vulnerabilidade }\end{array}$} & \multirow{3}{*}{ Fecundidade baixa } & V1 & 7373 & 97,02 & 0,0020 \\
\hline & & $\mathrm{V} 2$ & 7373 & 93,35 & 0,0029 \\
\hline & & V3 & 9618 & 69,64 & 0.0047 \\
\hline & Média & & & 86,67 & \\
\hline & \multirow{3}{*}{$\begin{array}{c}\text { Atenção e cuidados especiais } \\
\text { com crianças, adolescentes e } \\
\text { jovens }\end{array}$} & V4 & 9618 & 59,64 & 0.0050 \\
\hline & & V5 & 9618 & 41,45 & 0,0050 \\
\hline & & V6 & 9618 & 75,59 & 0,0044 \\
\hline & Média & & & 58,89 & \\
\hline & \multirow{2}{*}{ Dependência econômica } & V7 & 9618 & 63,40 & 0,0049 \\
\hline & & V8 & 9618 & 77,96 & 0,0042 \\
\hline & Média & & & 70,68 & \\
\hline & \multirow{2}{*}{ Presença da mãe } & V9 & 3515 & 98,83 & 0,0018 \\
\hline & & V10 & 3474 & 93,00 & 0,0043 \\
\hline & Média & & & 95,91 & \\
\hline Média 1 & & & & 74,05 & \\
\hline \multirow{9}{*}{$\begin{array}{l}\text { 2. Acesso ao } \\
\text { conhecimento }\end{array}$} & \multirow{2}{*}{ Ausência de analfabetismo } & $\mathrm{C} 1$ & 8580 & 99,14 & 0,0009 \\
\hline & & $\mathrm{C} 2$ & 8580 & 96,00 & 0,0021 \\
\hline & Média & & & $\mathbf{9 7 , 5 7}$ & \\
\hline & \multirow{2}{*}{ Escolaridade } & C3 & 8580 & 83,76 & 0,0039 \\
\hline & & $\mathrm{C} 4$ & 8580 & 67,53 & 0,0050 \\
\hline & Média & & & 75,64 & \\
\hline & \multirow{2}{*}{ Qualidade profissional } & C5 & 8580 & 26,70 & 0,0048 \\
\hline & & C6 & 7459 & 80,57 & 0,0046 \\
\hline & Média & & & 53,63 & \\
\hline Média 2 & & & & 74,69 & \\
\hline
\end{tabular}




\begin{tabular}{|c|c|c|c|c|c|}
\hline Dimensão & Componente & Indicadores & $\begin{array}{c}\text { Obs. } \\
\text { (Domicílios) }\end{array}$ & $\begin{array}{l}\text { Média } \\
(\%)\end{array}$ & $\begin{array}{l}\text { Desvio } \\
\text { Padráo }\end{array}$ \\
\hline \multirow{9}{*}{$\begin{array}{l}\text { 3. Acesso ao } \\
\text { trabalho }\end{array}$} & \multirow{2}{*}{ Disponibilidade de trabalho } & $\mathrm{T} 1$ & 9618 & 48,49 & 0,0051 \\
\hline & & $\mathrm{T} 2$ & 7508 & 95,38 & 0,0024 \\
\hline & Média & & & 71,93 & \\
\hline & \multirow{2}{*}{ Qualidade do posto de trabalho } & T3 & 9618 & 48,79 & 0,0051 \\
\hline & & $\mathrm{T} 4$ & 9618 & 77,99 & 0,0042 \\
\hline & Média & & & 63,39 & \\
\hline & \multirow{2}{*}{ Remuneração } & $\mathrm{T} 5$ & 9618 & 89,63 & 0,0031 \\
\hline & & $\mathrm{T} 6$ & 9618 & 65,19 & 0,0048 \\
\hline & Média & & & 77,41 & \\
\hline Média 3 & & & & 68,43 & \\
\hline \multirow{3}{*}{$\begin{array}{l}\text { 4. Disponibilidade } \\
\text { de Recursos }\end{array}$} & Não está em extrema pobreza & $\mathrm{R} 1$ & 9618 & 98,80 & 0,0011 \\
\hline & $\begin{array}{c}\text { Não está abaixo da linha de } \\
\text { pobreza }\end{array}$ & $\mathrm{R} 2$ & 9618 & 97,92 & 0,0014 \\
\hline & Capacidade de geração de renda & R3 & 9618 & 68,76 & 0,0047 \\
\hline Média 4 & & & & 88,49 & \\
\hline \multirow{15}{*}{$\begin{array}{c}5 . \\
\text { Desenvolvimento } \\
\text { Infantil }\end{array}$} & \multirow{2}{*}{ Trabalho precoce } & D1 & 3307 & 75,54 & 0,0074 \\
\hline & & D2 & 3702 & 77,90 & 0,0068 \\
\hline & Média & & & 76,72 & \\
\hline & \multirow{3}{*}{ Acesso à escola } & D3 & 1755 & 55,16 & 0,0119 \\
\hline & & D4 & 2181 & 99,54 & 0,0014 \\
\hline & & D5 & 2484 & 96,22 & 0,0035 \\
\hline & Média & & & 83,64 & \\
\hline & \multirow{3}{*}{ Progresso escolar } & D6 & 3307 & 97,49 & 0,0027 \\
\hline & & D7 & 1564 & 99,81 & 0,0011 \\
\hline & & D8 & 1145 & 99,39 & 0,0023 \\
\hline & Média & & & 98,89 & \\
\hline & \multirow{3}{*}{ Mortalidade Infantil } & D9 & 7373 & 90,56 & 0,0034 \\
\hline & & D10 & 7365 & 11,47 & 0,0037 \\
\hline & & D11 & 8723 & 97,87 & 0,0015 \\
\hline & Média & & & 66,63 & \\
\hline Média 5 & & & & 76,17 & \\
\hline
\end{tabular}




\begin{tabular}{|c|c|c|c|c|c|}
\hline Dimensão & Componente & Indicadores & \begin{tabular}{|c|}
$\begin{array}{c}\text { Obs. } \\
\text { (Domicílios) }\end{array}$ \\
\end{tabular} & $\begin{array}{c}\text { Média } \\
(\%)\end{array}$ & $\begin{array}{l}\text { Desvio } \\
\text { Padráo }\end{array}$ \\
\hline \multirow{14}{*}{$\begin{array}{l}\text { 6. Condiçóes } \\
\text { habitacionais }\end{array}$} & \multirow{2}{*}{ Propriedade } & $\mathrm{H} 1$ & 8776 & 76,17 & 0,0017 \\
\hline & & $\mathrm{H} 2$ & 9618 & 81,01 & 0,0039 \\
\hline & Média & & & 78,59 & \\
\hline & Déficit Habitacional & $\mathrm{H} 3$ & 9618 & 92,33 & 0,0027 \\
\hline & Abrigabilidade & $\mathrm{H} 4$ & 9618 & 82,48 & 0,0039 \\
\hline & Acesso a abastecimento de água & $\mathrm{H} 5$ & 9618 & 88,80 & 0,0032 \\
\hline & Acesso a saneamento & H6 & 9618 & 92,15 & 0,0027 \\
\hline & Acesso a coleta de lixo & $\mathrm{H} 7$ & 9618 & 96,05 & 0,0020 \\
\hline & Acesso a energia elétrica & $\mathrm{H} 8$ & 9618 & 99,74 & 0,0005 \\
\hline & \multirow{4}{*}{ Acesso a bens duráveis } & $\mathrm{H} 9$ & 9618 & 59,98 & 0,0051 \\
\hline & & $\mathrm{H} 10$ & 9618 & 49,86 & 0,0051 \\
\hline & & H11 & 9618 & 49,46 & 0,0051 \\
\hline & & $\mathrm{H} 12$ & 9618 & 33,90 & 0,0048 \\
\hline & Média & & & 74,47 & \\
\hline Média 6 & & & & 75,15 & \\
\hline
\end{tabular}

Fonte: Elaborado pelos autores com dados da PNAD - IBGE (2017).

É possível observar que no Estado do Rio Grande do Sul, as mulheres têm apresentado nos últimos anos (2014 e 2015) fecundidade baixa, em média 87\%. Em 2015 o Estado do Rio Grande do Sul possuía a segunda menor taxa de fecundidade $(1,56)$ entre as unidades federativas do Brasil, atrás apenas do Estado de Santa Catarina que obteve a taxa de fecundidade de 1,55 (IBGE, 2018). Além disso, evidencia-se que 69,6\% dos domicílios têm ausência de crianças, e quando estas estáo presentes, constata-se também a presença materna em 97\% dos casos. Com referência a escolaridade, a ausência de analfabetismo é de $97,6 \%$, mas a presença de pelo menos um adulto com educação superior ainda é de 26,7\% no Estado.

No que se refere ao mercado de trabalho o indicador T2 (Presença de pelo menos um trabalhador há mais de seis meses no trabalho atual) indica a baixa rotatividade no mercado de trabalho, o que pode estar relacionado à crise financeira no país e no Estado. No entanto, a presença de pelo menos um ocupado no setor formal (T3) foi de 48,8\% neste período.

Compreende-se que a crise financeira retrata a presença de 65,19\% nos domicílios de pelo menos um ocupado com rendimento superior a dois salários mínimos. Apesar dessa informação, 98\% das famílias não se encontram abaixo da linha de pobreza e conseguem ter alta capacidade de geração de renda. Em outras palavras, mesmo com 48,8\% dos domicílios com pelo menos uma pessoa no setor formal, as famílias buscam gerar renda por conta própria.

O Desenvolvimento Infantil é destacado nos resultados, já que existe um considerável acesso e progresso escolar (84\% e 99\%, respectivamente) e ausência de mãe com filho nascido morto (97,87\%). As condiçóes habitacionais no Estado também são positivas, com $92 \%$ de acesso ao saneamento básico e 99,7\% dos domicílios com acesso à energia elétrica. 
O acesso a bens duráveis tem uma média de 33,90 quando o computador é inserido neste item.

De acordo com a abordagem de Barros, Carvalho e Franco (2003), através de uma média simples obteve-se os seguintes índices (TABELA 2).

Tabela 2 - IDF $_{\text {Barros }}$ por dimensão e IDF $_{\text {Barros }}$ das famílias do estado do Rio Grande do Sul para o ano de 2015

\begin{tabular}{l|c}
\hline \multicolumn{1}{c|}{ Dimensáo } & Índice \\
\hline 1. Ausência de vulnerabilidade & 74,05 \\
\hline 2. Acesso ao conhecimento & 74,69 \\
\hline 3. Acesso ao trabalho & 68,43 \\
\hline 4. Disponibilidade de Recursos & 88,49 \\
\hline 5. Desenvolvimento Infantil & 76,17 \\
\hline 6. Condiçóes habitacionais & 74,47 \\
\hline Índice de Desenvolvimento das Famílias - IDF $_{\text {Barros }}$ & $\mathbf{7 5 , 8 4}$ \\
\hline
\end{tabular}

Fonte: Resultados da pesquisa.

A Tabela 2 expõe um resumo dos indicadores apresentados na Tabela 1, demonstrando seis dimensóes. Classificando o índice de 80 a 100 como muito alto, de 70 a 79,99 alto, de 60 a 69,99 médio, de 50 a 59,99 baixo e 0 a 49,99 baixo ${ }^{11}$, verifica-se que os maiores índices foram obtidos nas dimensões de Disponibilidade de Recursos e o Desenvolvimento Infantil nos domicílios do Estado do Rio Grande do Sul, em que apresentaram muito alto e alto $\mathrm{IDF}_{\text {Barros }}$ respectivamente, o mesmo náo ocorre para a dimensão de Acesso ao Trabalho que obteve médio $\mathrm{IDF}_{\text {Barros. }}$. Assim, o Estado tem como índice de Desenvolvimento das Famílias $\left(\mathrm{IDF}_{\mathrm{Barros}}\right)$ um valor de 75,84, podendo ser declarado como alto desenvolvimento. O mesmo cálculo realizado para o Brasil gerou um IDF de 73,52, ou seja, em média as famílias no Estado do Rio Grande do Sul estão com IDF acima da média nacional. Na próxima seção estes indicadores são analisados pelo método de análise fatorial.

\subsubsection{Análise Fatorial}

Primeiro foi realizada uma análise de correlação para averiguar a fatorabilidade, ou seja, se as variáveis têm variância comum. Logo, foi observado que algumas variáveis apresentaram altas correlaçóes entre si (colinearidade), não sendo possível o cálculo das variâncias para a estimação do modelo. Esse problema pode estar ocorrendo porque as variáveis são dicotômicas. Para estimaçóes de Análise Fatorial para variáveis binárias,

11 Classificação elaborada com base no Índice de Desenvolvimento Humano Municipal (ATLAS DO DESENVOLVIMENTO HUMANO NO BRASIL - 2018). 
existem algumas recomendações de como usar correlaçóes tetracóricas ${ }^{12}$ ao invés de Pearson. Também é recomendável supor as variáveis dicotômicas como subjacentes contínuas ${ }^{13}$ ou usar a Teoria da resposta do item, em vez da Análise Fatorial.

Assim, algumas variáveis precisaram ser excluídas (V4, V5. V9, V10, T5, D3, D7, D8 e D10), por causa dos problemas de colinearidade e de variabilidade. Foi também decidido manter os missing no banco de dados, para não ocorrer a perda de informação.

Também foi testada a confiabilidade da Estrutura Fatorial utilizando o critério do Alpha de Cronbach ${ }^{14}$. Este avalia o grau das variáveis que tenham consistência interna de uma matriz que estão correlacionadas entre si. O resultado foi 0,75 , estando dentro de um intervalo aceitável. Para o caso de variáveis binárias foi analisada a matriz de correlação tetracórica ${ }^{15}$ também não apresentou missing e gerou boas correlações.

Logo, foi observada a Análise Fatorial Exploratória por correlação de Pearson e por correlação tetracórica. As duas matrizes geraram resultados diferentes, a matriz de correlação tetracórica analisou mais as observaçôes e apresentou os melhores resultados ${ }^{16}$, por essa razão continuou a análise pela referida matriz. Já na Análise Fatorial Exploratória foi realizada com o método de componentes principais e com uma rotação ortogonal - varimax.

Assim dos 39 indicadores gerou-se 10 fatores com autovalores acima de um, mas optou-se por usar as seis primeiras dimensóes, para ter coerência com o trabalho de Barros, Carvalho e Franco (2003). Estes autores atribuíram 69\% da variabilidade das variáveis, conforme mostra a Tabela 3.

Tabela 3: Resultado da análise Fatorial Exploratória

\begin{tabular}{l|l|l|l}
\hline Fator & Variância & Proporçáo & Cumulado \\
\hline Fator1 & 5,27141 & 0,1352 & 0,1352 \\
\hline Fator2 & 4,57682 & 0,1174 & 0,2525 \\
\hline Fator3 & 4,36039 & 0,1118 & 0,3643 \\
\hline Fator4 & 3,66614 & 0,0940 & 0,4583 \\
\hline Fator5 & 3,44426 & 0,0883 & 0,5466 \\
\hline
\end{tabular}

12 A correlação tetracórica é usada quando ambas as variáveis que estão correlacionadas são binárias, caso semelhante da correlação de Pearson, sendo que essa estima as correlaçóes para variáveis continuas (UEBERSAX, 2000). Para mais informaçóes sobre a correlação tetracórica conferir os trabalhos de Drasgow (1988) e de Harris (1988).

13 Elas representam uma quase-variável continua artificialmente dicotomizada.

14 Como pode ter itens que não são da mesma escala, foi analisado com base em variáveis padronizadas.

15 A matriz de correlação tetracórica foi realizada com a análise pairwise para poder ser utilizada para a análise fatorial ou por componentes principais de variáveis binárias conforme Stata Manual (2015).

16 Foi averiguado o critério KMO (Kaiser-Meyer-Olkin) para a Análise Fatorial com correlação de Pearson e obteve-se o resultado de 0,98 . 


\begin{tabular}{l|l|l|l}
\hline Fator & Variância & Proporçáo & Cumulado \\
\hline Fator6 & 3,31100 & 0,0849 & 0,6315 \\
\hline Fator7 & 2,79805 & 0,0717 & 0,7033 \\
\hline Fator8 & 2,44243 & 0,0626 & 0,7659 \\
\hline Fator9 & 1,63792 & 0,0420 & 0,8079 \\
\hline Fator10 & 1,16491 & 0,0299 & 0,8378 \\
\hline
\end{tabular}

LR test: independent vs, saturated: $\operatorname{chi} 2(741)=3,4 \mathrm{e}+06$ Prob $>$ chi $2=0,0000$

Fonte: Resultados da pesquisa.

Para determinar os fatores, a Tabela 4 apresenta as cargas fatoriais, observou-se que o fator 01 apresentou cargas altas e positivas nas variáveis C1 (ausência de adulto analfabeto), C2 (ausência de adulto analfabeto funcional), C3 (presença de pelo menos um adulto com fundamental completo), C4 (presença de pelo menos um adulto com ensino médio completo), C5 (Presença de pelo menos um adulto com alguma educação superior) e C6 (presença de pelo menos um trabalhador com qualificação média ou alta), podendo representar a dimensão “Acesso ao Conhecimento”. Já o fator 02 apresentou cargas fatoriais positivas para as variáveis V1 (nenhuma mulher teve filho nascido vivo no último ano), V2 (nenhuma mulher teve filho nascido vivo nos últimos dois anos), V3 (Ausência de criança), H3 (Densidade de até dois moradores por dormitório), podendo esse fator ser chamado de "Ausência de Vulnerabilidade".

Tabela 4 - Cargas fatoriais da Análise Fatorial Exploratória

\begin{tabular}{c|c|c|l|l|l|c|c}
\hline Variável & Fator1 & Fator2 & Fator3 & Fator4 & Fator5 & Fator6 & Comunalidade \\
\hline V1 & & 0,7582 & & & & & 0,8574 \\
\hline V2 & & 0,8562 & & & & & 0,8532 \\
\hline V3 & & 0,9001 & & & & & 0,8826 \\
\hline V6 & & $-0,3060$ & & & & 0,8744 & 0,951 \\
\hline V7 & & $-0,4327$ & & & & & 0,5044 \\
\hline V8 & & & & & & 0,9359 & 0,9171 \\
\hline C1 & 0,6414 & & & & & & 0,9709 \\
\hline C2 & 0,8603 & & & & & & 0,9144 \\
\hline C3 & 0,9190 & & & & & & 0,9453 \\
\hline C4 & 0,9482 & & & & & & 0,9756 \\
\hline C5 & 0,9175 & & & & & & 0,9091 \\
\hline C6 & 0,8081 & & & & & & 0,7989 \\
\hline T1 & & & & & 0,7048 & 0,4132 & 0,7796 \\
\hline T2 & & & & & & & 0,7765 \\
\hline T3 & & & & & 0,7656 & 0,3281 & 0,8348 \\
\hline T4 & 0,3096 & $-0,3088$ & & & 0,4196 & 0,5726 & 0,87 \\
\hline T6 & 0,3720 & $-0,6554$ & & & & & 0,7016 \\
\hline
\end{tabular}




\begin{tabular}{c|l|l|l|l|l|l|c}
\hline Variável & Fator1 & Fator2 & Fator3 & Fator4 & Fator5 & Fator6 & Comunalidade \\
\hline R1 & & & & & 0,9468 & & 0,9604 \\
\hline R2 & & & & & 0,8106 & & 0,8802 \\
\hline R3 & & $-0,3452$ & & & 0,5938 & 0,5984 & 0,893 \\
\hline H1 & & & & & & & 0,8826 \\
\hline H2 & & & & & & & 0,9123 \\
\hline H3 & & 0,6635 & & & & & 0,7574 \\
\hline H4 & & & & & & & 0,4189 \\
\hline H5 & & & & & & & 0,6869 \\
\hline H6 & & & & & & & 0,5962 \\
\hline H7 & & & 0,3387 & & & & 0,8763 \\
\hline H8 & & & 0,3389 & & & & 0,9424 \\
\hline H9 & & & 0,9854 & & & & 0,9947 \\
\hline H10 & & & 0,9855 & & & & 0,9946 \\
\hline H11 & & & 0,9821 & & & & 0,9922 \\
\hline H12 & & & 0,8885 & & & & 0,977 \\
\hline D1 & & 0,3595 & & 0,8866 & & & 0,9322 \\
\hline D2 & & 0,3189 & & 0,9208 & & & 0,9709 \\
\hline D4 & & & & 0,9004 & & & 0,9565 \\
\hline D5 & & & & 0,9052 & & & 0,9723 \\
\hline D6 & & $-0,5405$ & & & & & 0,7997 \\
\hline D9 & & & & & & 0,3666 & 0,3757 \\
\hline D11 & & & & & & 0,4585 \\
\hline
\end{tabular}

Fonte: Resultados do estudo.

O fator 03 pode ser denominado de "Condições Habitacionais", pois a variabilidade das variáveis H7 (lixo é coletado), H8 (acesso a eletricidade), H9 (acesso a fogão e geladeira), H10 (acesso a fogão, geladeira, televisão ou rádio), H11 (acesso a fogão, geladeira, televisão ou rádio e telefone) e H12 (acesso a fogão, geladeira, televisão ou rádio, telefone e computador) são atribuídas a esse fator comum. O fator 04 tem cargas positivas nas variáveis D1 (ausência de criança com menos de 14 anos trabalhando), D2 (ausência de criança com menos de 16 anos trabalhando), D4 (ausência de criança de 07 a 14 anos fora da escola), D5 (ausência de criança de 07 a 17 anos fora da escola), podendo ser identificado como "Desenvolvimento Infantil".

O fator 05 tem altas cargas, principalmente nas variáveis R1 (renda familiar per capita superior à linha de extrema pobreza) e R2 (renda familiar per capita superior à linha de pobreza). Logo, as variáveis T1 (mais da metade dos membros em idade ativa encontrase ocupada) e T3 (presença de pelo menos um ocupado no setor formal) e, posteriormente, a R3 (maior parte da renda não advém de transferências) observa-se uma correlação da disponibilidade de recursos e do acesso ao trabalho, mas pelas cargas serem maiores nas 
variáveis R1 e R2 denomina-se a dimensão como "Disponibilidade de recurso". Já no fator 6 observa-se uma correlação com variáveis de acesso ao trabalho, disponibilidade de recursos e idade ativa pelas variáveis V3 (ausência de idoso) e V8 (mais da metade dos membros encontra-se em idade ativa), portanto o fator 6 pode ser denominado "Acesso ao Trabalho". Em razáo do que foi apresentado, averiguou-se uma similaridade com o modelo proposto por Barros Carvalho e Franco (2003).

\subsection{2 Índice pela Análise Fatorial - IDF ${ }_{\text {Fatorial }}$}

Para alcançar o terceiro e o quarto objetivo específico proposto, foi calculado o $\mathrm{IDF}_{\text {Fatorial }}$ e comparado com o IDF $\mathrm{Barros}$. Através da Análise Fatorial Exploratória foram calculados os escores e os índices respectivos.

O Índice de Desenvolvimento das Famílias do Rio Grande do Sul ( $\left.\operatorname{IDF}_{\text {Fatorial }}\right)$ foi de 56,83. Já o $\operatorname{IDF}_{\text {Barros }}$ obtido pela média das variáveis apresentou um valor de 75,84, ou seja, sem o método de análise multivariada as médias sobre-estimam o índice. $\mathrm{O}$ mesmo cálculo do $\mathrm{IDF}_{\text {Fatorial }}$ para o Brasil foi realizado com o mesmo número de fatores e obteve-se um índice de 57,31.

Vale ressaltar que os métodos utilizam formas diferentes para ponderar o cálculo do índice, sendo que pela Análise Fatorial Exploratória as variáveis que mais representam um determinado fator possuem maior peso, podendo ser uma estimativa melhor.

\subsubsection{Análise de Cluster}

Para realizar a análise de clusters do $\mathrm{IDF}_{\text {Fatorial, }}$ no sentido de identificar grupos homogêneos de domicílios com condiçóes de vida semelhantes, utilizou-se os escores fatoriais obtidos da Análise Fatorial Exploratória. Para identificar o número de grupos, estabeleceuse primeiro analisar pelo método de cluster hierárquico aglomerativo, com intuito de se obter o número de cluster mais apropriado pelo índice de Duda-Hart e pela estatística Pseudo $\mathrm{T}^{2}$. Logo, foram identificados 15 grupos, conforme evidenciado na Tabela 5.

Tabela 5 - Número de Cluster

\begin{tabular}{c|c|c}
\hline \multirow{2}{*}{ Número de Cluster } & \multirow{2}{*}{$(\mathbf{2}) / \mathbf{J e}(\mathbf{1})$} & Pseudo \\
\cline { 3 - 3 } & 0,7259 & T-squared \\
\hline 1 & 0,6992 & 1064,48 \\
\hline 2 & 0,7682 & 629,84 \\
\hline 3 & 0,6378 & 408,27 \\
\hline 5 & 0,7752 & 302,12 \\
\hline 6 & 0,7916 & 285,03 \\
\hline 7 & 0,7132 & 215,65 \\
\hline 8 & 0,7105 & 203,08 \\
\hline 9 & 0,7543 & 195,16 \\
\hline
\end{tabular}




\begin{tabular}{c|c|c}
\hline \multirow{2}{*}{ Número de Cluster } & \multirow{2}{*}{$(\mathbf{2}) / \mathbf{J e}(\mathbf{1})$} & Pseudo \\
\cline { 3 - 3 } & 0,8361 & T-squared \\
\hline 10 & 0,5966 & 93,31 \\
\hline 11 & 0,5700 & 248,84 \\
\hline 12 & 0,7021 & 141,05 \\
\hline 13 & 0,7262 & 118,36 \\
\hline 14 & 0,3092 & 120,67 \\
\hline 15 & & 429,01 \\
\hline
\end{tabular}

Fonte: Resultados da análise.

Desta informação de 15 grupos, foi realizado o método de cluster não hierárquico (k-médias), em virtude de ser o mais indicado para um número grande de observaçóes, com distância Euclidiana para a medida de similaridade. Obteve-se os seguintes resultados, conforme mostrado na Tabela 6.

Tabela 6 - Estatística Cluster Final: frequência e média

\begin{tabular}{c|c|c|c|c|c|c|c}
\hline \multirow{2}{*}{ Cluster } & \multirow{2}{*}{ Frequência } & \multicolumn{7}{|c}{ Média } \\
\cline { 3 - 8 } & & F1 & F2 & F3 & F4 & F5 & F6 \\
\hline 1 & 308 & 0,077349 & 0,308941 & 0,765477 & 0,940244 & 0,704854 & 0,991068 \\
\hline 2 & 113 & 0,171736 & 0,320491 & $-0,24722$ & $-0,039909$ & 0,852873 & $1,203,488$ \\
\hline 3 & 202 & 0,160805 & 0,467076 & 0,833304 & 0,861884 & 0,048311 & $1,173,113$ \\
\hline 4 & 274 & 0,624468 & 0,378196 & 0,828641 & 0,970695 & 0,501473 & 0,737087 \\
\hline 5 & 209 & 0,185314 & 0,490857 & $-0,169077$ & 0,876854 & 0,110796 & $1,245,922$ \\
\hline 6 & 152 & 0,032885 & 0,179880 & 0,737645 & 0,522787 & $1,094,165$ & 0,315176 \\
\hline 7 & 81 & 0,269340 & 0,151432 & $-0,226556$ & 0,871872 & $1,093,037$ & 0,046685 \\
\hline 8 & 353 & 0,124291 & 0,201289 & 0,798182 & $-0,080298$ & 0,603632 & 0,957609 \\
\hline 9 & 269 & $-0,227773$ & 0,178211 & $-0,109206$ & $1,002,90$ & 0,642247 & 0,845134 \\
\hline 10 & 177 & $-0,340787$ & 0,201064 & 0,919953 & 0,989379 & 0,679810 & 0,724064 \\
\hline 11 & 74 & $-0,044600$ & 0,069998 & $-0,186629$ & $-0,073500$ & 0,151452 & $1,102,594$ \\
\hline 12 & 127 & $-0,181226$ & 0,144095 & $-0,222991$ & 0,233636 & $1,061,623$ & 0,607333 \\
\hline 13 & 101 & 0,405060 & 0,014735 & $-0,271280$ & 0,009536 & 0,566303 & 0,830364 \\
\hline 14 & 135 & 0,754281 & 0,401039 & $-0,202635$ & $1,006,39$ & 0,477880 & 0,778230 \\
\hline 15 & 246 & 0,258091 & 0,251626 & $-0,286782$ & 0,965893 & 0,72616 & $1,028,523$ \\
\hline
\end{tabular}

Fonte: Resultados da pesquisa.

A Tabela 6 mostra a frequência de domicílios e a média dos escores fatoriais em cada grupo. Observou-se que o grupo 01 é o que mais tem domicílios, sendo similar com o perfil F6 (Acesso ao trabalho) e mais distante do F1 (Acesso ao conhecimento), mas todos os fatores obtiveram valores positivos. Por sua vez, o grupo 11 tem 74 domicílios, sendo mais próximo do F6 (Acesso ao trabalho) e mais afastado de F3 (condiçóes habitacionais). 
O grupo 07, com 81 domicílios, está mais longe do acesso de trabalho (F6) e mais similar com disponibilidade de recurso. Este último resultado pode ser explicado por famílias que não tem as características de acesso de trabalho, como a presença de pelo menos um ocupado no setor formal. Por outro lado, possuem características de disponibilidade de recursos, pois podem ser famílias que trabalham na área rural, por exemplo.

Foi oportuno observar algumas características dentro de cada grupo, como a presença de idosos, de negros ${ }^{17}$, mulher como chefe da família ${ }^{18}$, da área rural, da presença de pelo menos um adulto com ensino médio completo, da presença de pelo menos um ocupado no setor formal e do Índice de Desenvolvimento Familiar por grupo, conforme Tabela 7.

Tabela 7 - Grupos e determinadas características das famílias no Rio Grande do Sul em 2015

\begin{tabular}{c|c|c|c|c|c|c|c|c}
\hline Grupo & Domicílios & $\begin{array}{c}\text { Idoso } \\
\mathbf{( \% )}\end{array}$ & $\begin{array}{c}\text { Pres. pelo } \\
\text { menos } \\
\text { uma pessoa } \\
\text { negra (\%) }\end{array}$ & $\begin{array}{c}\text { Chefe } \\
\text { domiciliar } \\
\text { mulher } \\
\mathbf{( \% )}\end{array}$ & $\begin{array}{c}\text { Área } \\
\text { Rural } \\
\mathbf{( \% )}\end{array}$ & $\begin{array}{c}\text { Pres. pelo } \\
\text { menos 1 } \\
\text { adulto ensino } \\
\text { med. completo } \\
\text { (\%) }\end{array}$ & $\begin{array}{c}\text { Pres. pelo } \\
\text { menos 1 } \\
\text { ocup. setor } \\
\text { formal (\%) }\end{array}$ & $\begin{array}{c}\text { IDF } \\
\mathbf{( \% )}\end{array}$ \\
\hline 1 & 308 & 0,3 & 16,88 & 37,34 & 3,25 & 91,55 & 99,33 & 61,59 \\
\hline 2 & 113 & 0,00 & 14,16 & 35,40 & 13,27 & 89,38 & 92,92 & 43,25 \\
\hline 3 & 202 & 0,50 & 8,42 & 34,65 & 11,88 & 84,65 & 17,82 & 62,12 \\
\hline 4 & 274 & 7,66 & 6,90 & 39,78 & 2,92 & 100,00 & 60,21 & 67,64 \\
\hline 5 & 209 & 0,90 & 16,74 & 40,19 & 20,09 & 66,98 & 22,97 & 52,19 \\
\hline 6 & 152 & 36,84 & 20,39 & 37,50 & 3,28 & 84,21 & 90,13 & 53,40 \\
\hline 7 & 81 & 90,12 & 14,81 & 51,85 & 18,52 & 69,13 & 71,60 & 47,08 \\
\hline 8 & 353 & 0,84 & 9,35 & 40,51 & 4,53 & 85,83 & 67,43 & 50,11 \\
\hline 9 & 269 & 1,49 & 13,38 & 40,89 & 20,45 & 1,86 & 60,22 & 45,98 \\
\hline 10 & 177 & 4,52 & 14,68 & 44,63 & 6,77 & 2,25 & 70,62 & 56,03 \\
\hline 11 & 74 & 1,35 & 10,81 & 36,49 & 20,27 & 58,11 & 12,16 & 33,81 \\
\hline 12 & 127 & 11,02 & 21,25 & 35,43 & 6,30 & 38,58 & 84,25 & 37,91 \\
\hline 13 & 101 & 4,95 & 16,83 & 46,53 & 4,95 & 99,00 & 68,31 & 39,06 \\
\hline 14 & 135 & 11,85 & 13,33 & 36,29 & 8,15 & 99,25 & 59,26 & 58,22 \\
\hline 15 & 246 & 0,00 & 15,04 & 36,99 & 7,31 & 96,75 & 99,59 & 51,65 \\
\hline
\end{tabular}

Fonte: Resultados da pesquisa.

Nos resultados apresentados percebe-se que grupos com maior densidade de domicílios na área rural tendem a possuir um IDF menor como nos grupos 03, 05, 07, 09 e 11. Verifica-se também nos últimos grupos que a percentagem de domicílios com pelos

17 Na Pesquisa Nacional por Amostra de Domicílios, a raça/cor são autodeclaradas pelos entrevistados.

18 Chefe de família é definido como pessoa de referência na família, respondida pelo entrevistado na Pesquisa Nacional por Amostra de Domicílios. 
menos um adulto com ensino médio completo é menor que em outros grupos. Além disso, esses grupos possuem menor presença de um adulto no setor formal. O grupo 07, no qual observou-se a maior concentração de idosos, obteve IDF mais baixo que os outros grupos, de 47,08. Ressalta-se que quase 19\% dos domicílios se encontrava em área rural.

A presença de pelo menos uma pessoa negra no domicílio foi, em média, muito semelhante entre os grupos, não apresentando disparidade. Entretanto, averiguou-se que o IDF mais alto de todos os clusters foi no grupo 04, com IDF de 67,64. Constata-se que $100 \%$ dos domicílios tinham pelo menos um adulto com ensino médio completo, mas foi o grupo com menor densidade de domicílios com a presença de pelo menos uma pessoa negra. Tal situação indica uma desigualdade racial no que diz respeito ao grau de escolarização das famílias investigadas, bem como uma possível correlação entre escolaridade e a qualidade de vida. Em outras palavras, ainda há dificuldades para pessoas de raça negra terem melhores condições de vida no estado do Rio Grande do Sul.

O interessante foi verificar que o grupo com maior IDF (grupo 04 com IDF 67,64) teve a maior percentagem de domicílios com a presença de pelo menos um adulto com ensino médio completo (100\%). Porém, não necessariamente este grupo obteve a maior percentagem entre os grupos de domicílios com a presença de pelo menos um adulto no setor formal, demonstrando assim a importância de ser empreendedor ou autônomo.

Ao mesmo tempo, o grupo 01 demonstra que a maioria dos domicílios com pelo menos um adulto com ensino médio completo e pelo menos um adulto no setor formal, também apresentam alto IDF $(61,59)$. Os grupos que possuem poucos domicílios com baixa presença de um adulto no setor formal, apresentaram baixo IDF $(33,83)$, como demonstrado no grupo 11 .

A questão de gênero com referência ao chefe de família parece não afetar muito o $\mathrm{IDF}_{\text {Fatorial' }}$, pois há grupos com maior percentagem de chefe de família mulher sem apresentar um grande diferencial no IDF, embora seja perceptível que na maioria dos grupos o homem é quem assume o papel da chefia familiar. Apenas o grupo 07 que detém mais da metade dos domicílios com chefe de família mulher, considerado um grupo muito pequeno, com apenas 80 domicílios.

Por fim, foi realizado agrupamentos pelo índice criado pela Análise Fatorial, também por cluster não hierárquico com distância Euclidiana em três grupos (TABELA 8).

Tabela 8 - Estatísticas sumárias por categoria de cluster

\begin{tabular}{c|c|c|c|c}
\hline Grupo - IDF & Frequência & Mínimo & Média & Máximo \\
\hline 1 & 675 & 23,61 & 39,90 & 45.79 \\
\hline 2 & 1145 & 45.82 & 51,69 & 57,53 \\
\hline 3 & 1001 & 57.57 & 63.41 & 79.07 \\
\hline Total & 2821 & 23,61 & 53,03 & 79,07 \\
\hline
\end{tabular}

Fonte: Resultados da pesquisa. 
A Tabela 8 mostra que 675 famílias (domicílios) estão no grupo 01, com intervalo de índice de 23,61 a 45,79, podendo estar classificado como baixo desenvolvimento familiar. No grupo 02, observa-se 1145 famílias com intervalo de índice de 45,82 a 57,53 estando num intervalo de médio desenvolvimento familiar. Também verifica-se 1001 domicílios com intervalo de índice de 57,51 a 79,07, situando-se em alto desenvolvimento familiar. Os resultados demonstram que existe um grande gap de 79,07 a 100, mostrando que nenhuma família está conseguindo alcançar um alto valor do IDF.

\section{CONCLUSÃO}

Este estudo buscou identificar o Índice de Desenvolvimento das Famílias do Estado do Rio Grande do Sul para o ano de 2015, bem como as dimensóes latentes que representam o conjunto de variáveis analisadas. Também almejou-se agrupar famílias por grau de semelhança dos escores fatoriais.

Os indicadores evidenciaram que no referido estado, apesar de atravessar uma crise econômica, as famílias possuem grande capacidade de geração de recursos, estando acima da linha da pobreza. A quantidade de domicílios que tinham ausência de mortalidade infantil foi elevada, como também o acesso e o progresso escolar. A baixa fecundidade das mulheres foi observada no Estado para os últimos anos, por conseguinte, 69,6\% dos domicílios apresentaram ausência de crianças, demonstrando um novo perfil das famílias investigadas.

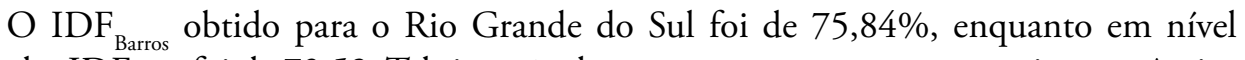
nacional o IDF ${ }_{\text {Barros }}$ foi de 73,52. Tal situaçâo demonstra que mesmo com a crise econômica do Rio Grande do Sul as famílias estariam com um IDF acima da média nacional, porém o mesmo resultado não é obtido pela Análise Fatorial.

O estudo pela Análise Multivariada, com a técnica de Análise Fatorial, gerou seis dimensóes latentes que explicam $69 \%$ da variabilidade dos indicadores. O Îndice de Desenvolvimento Familiar (IDF $\mathrm{Iatorial}_{\text {) }}$ obtido pelos escores destas dimensóes, foi de 56,83\%.

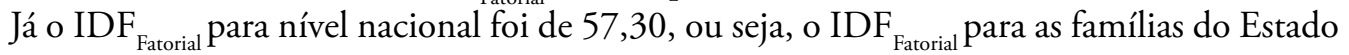
do Rio Grande do Sul estariam um pouco abaixo da média nacional.

Apesar dos diferentes resultados entre o $\operatorname{IDF}_{\text {Barros }}$ e o $\operatorname{IDF}_{\text {Fatorial, }}$ conclui-se que ambas estáo perto da média nacional, portanto as famílias do Estado do Rio Grande do Sul estão conseguindo acompanhar o bem-estar médio do país. Salienta-se que o Brasil também sofre com uma crise financeira e política, mas o Estado do Rio Grande do Sul foi o segundo Estado a decretar estado de calamidade financeira em 2016 dentre os estados de Rio de Janeiro e Minas Gerais, por isso, o interesse de observar como o IDF do Estado seguia o IDF nacional para o ano de 2015.

Pela análise de cluster, obteve-se 15 grupos, observou-se que a escolaridade, o setor formal e o empreendedor, são fatores que influenciam o maior IDF. Por outro lado, os grupos com maior densidade de domićlios na área rural, bem como a presença de idosos impactam na diminuição do IDF. Tal verificação deve chegar ao conhecimento das autoridades políticas, no sentido de fomentar programas sociais na área da educação, da saúde e da segurança, principalmente para os grupos de famílias com menor IDF. 
Uma extensão deste estudo pode ser identificar, através dos estratos da PNAD, onde estão localizadas os grupos de domicílios, uma vez que o estado possui Conselhos Regionais de Desenvolvimento (COREDEs), na qual autoridades buscam discutir e analisar a situaçáo socioeconômicas das 28 regióes que compóe o estado do Rio Grande do Sul. Assim, seria interessante comparar a situação econômica das regióes com as encontradas pelo IDF. Além disso, seria possível também comparar com o IDF de outros anos, a fim de verificar um acompanhamento de uma série histórica.

\section{REFERÊNCIAS}

ALMEIDA, A. C. A qualidade de vida no Estado do Rio de Janeiro. Niterói: Eduff, 1997.

ATLAS DO DESENVOLVIMENTO HUMANO NO BRASIL. O IDHM. Disponível em: < http://www.atlasbrasil.org.br/2013/pt/o_atlas/idhm/>. Acesso em: 05/02/2018.

\section{BARROS, R. P.; CARVALHO, M.; FRANCO S. O Índice de Desenvolvimento da} Família (IDF). Texto para discussão n ${ }^{\circ}$ 986. IPEA. 2003.

BECKER, G. S. A Theory of the Allocation of Time. The economic journal, p. 493-517, 1965.

BECKER, G. S.; TOMES, N. Human capital and the rise and fall of families. Journal of labor economics, v. 4, n. 3, Part 2, p. S1-S39, 1986.

BOURGUIGNON, F.; CHAKRAVARTY, S. R. The measurement of multidimensional poverty. Journal of Economic Inequality, v. 1, n.1, p. 25-49, 2003.

BRASIL. Decreto no 8.262, DE 31 DE MAIO DE 2014. Altera o Decreto no 2.018, de $1^{\circ}$ de outubro de 1996, que regulamenta a Lei no 9.294, de 15 de julho de 1996.

LEI No 8.069, DE 13 DE JULHO DE 1990. Dispóe sobre o Estatuto da Criança e do Adolescente e dá outras providências.

. LEI No 10.741, DE 1 DE OUTUBRO DE 2003. Dispóe sobre o Estatuto do Idoso e dá outras providências.

CHAKRAVARTY, S. R.; MUKHERJEE, D.; RANADE, R. On the family of subgroup and factor decomposable measures of multidimensional poverty. Research on Economic Inequality, v. 8, p. 175-94, 1998.

CIDE. Fundação Centro de Informações e Dados do Rio de Janeiro. IQM — Índice de Qualidade dos Municípios, 1998. Rio de Janeiro, 1998. 
CONGER, R. D.; CONGER, K. J.; ELDER, G. H.; LORENZ, F. O.; SIMONS, R. L.; WHITBECK, L. B. A family process model of economic hardship and adjustment of early adolescent boys. Child development, v. 63, n. 3, p. 526-541, 1992.

DE SOUZA, Nali de Jesus. Economia regional: conceito e fundamentos teóricos. 1981.

DOWBOR, L. A economia da família. Psicologia USP, v. 26, n. 1, 2015.

DRASGOW, F. Polychoric and polyserial correlations. In Kotz L, Johnson NL (Eds.), Encyclopedia of statistical sciences. V. 7 (pp. 69-74). New York: Wiley, 1988.

FALCÃO, T.; COSTA, P. V. A linha de extrema pobreza e público alvo do Plano Brasil sem miséria. IN: O Brasil sem miséria. Ministério do Desenvolvimento Social e Combate à fome. $1^{\text {a }}$ ediçãao. Brasília. 2014.

FEE - Fundação de Economia e Estatística Siegfried Emanuel Heuser. Índice de Desenvolvimento Socioeconômico (Idese). Disponível em: < http://www.fee.rs.gov.br/ indicadores/indice-de-desenvolvimento-socioeconomico/> Acesso em: 12/03/2013.

FERREIRA, M. C. T.; MARTURANO, E. M. Ambiente familiar e os problemas do comportamento apresentados por crianças com baixo desempenho escolar. Psicologia: Reflexáo e crítica, v. 15, n. 1, p. 35-44, 2002.

GUIMARÃES, E. N. Economia regional: elementos conceituais e metodológicos. 1997.

HAIR, J. F.; BLACK, W. C.; BABIN, B. J. ANDERSON, R. E. Multivariate Data Analysis. Prentice Hall; $7^{\text {a }}$ Edição. 785 p.

HARRIS, B. Tetrachoric correlation coefficient. In Kotz L, Johnson NL (Eds.), Encyclopedia of statistical sciences. V. 9 (pp. 223-225). New York: Wiley, 1988.

HOFFMANN, R.; KAGEYAMA, A. Pobreza no Brasil: uma perspectiva multidimensional. Revista Economia e Sociedade, Campinas, v.15, n.1 (26), p. 79-112. 2006.

IBGE - INSTITUTO BRASILEIRO DE GEOGRAFIA E ESTATÍSTICA. Indicadores de Desenvolvimento Sustentável - Tabela 3727 - Taxa de fecundidade total. SIDRA. Disponível em: < https://sidra.ibge.gov.br/tabela/3727>. Acesso em: 16/02/2018.

IBGE - INSTITUTO BRASILEIRO DE GEOGRAFIA E ESTATÍSTICA. PNAD - Pesquisa Nacional por Amostras de Domicílios do ano de 2015. Disponível em: <https://ww2.ibge.gov.br/home/estatistica/populacao/trabalhoerendimento/pnad2015/ microdados.shtm> Acesso em: 15/02/2017. 
IBGE - INSTITUTO BRASILEIRO DE GEOGRAFIA E ESTATÍSTICA. Tábua completa de mortalidade para o Brasil - 2015. Disponível em: <ftp://ftp.ibge.gov.br/ Tabuas_Completas_de_Mortalidade/Tabuas_Completas_de_Mortalidade_2015/tabua_ de_mortalidade_analise.pdf>. Acesso em: 16/02/2018.

LIMA, J. E. Curso de Análise Estatística Multivariada. Apostila. Departamento de Economia Rural. Universidade Federal de Viçosa. 2017.

MINGOTI, S. A. Análise de dados através de métodos de estatística multivariada. Uma abordagem aplicada. Editora UFMG. Belo Horizonte. 2013.

MOREIRA, M. A. Impactos da crise financeira do Rio Grande do Sul sobre o investimento em saúde pública nos anos 2000. Fundação de Economia e Estatística Siegfried Emanuel Heuser. Textos para Discussão FEE Nº 121. 2014.

OLIVEIRA, L. L. S. Idese e Indicadores Sociais: Origens e Evolução. Fundação de Economia e Estatística Siegfried Emanuel Heuser. Textos para Discussão FEE Nº 116. 2013.

PEARCE, J. Social Enterprise in Any town. London, Calouste Gulbenkian Foundation. 2003. 144 p.

ROCHA, S. Do consumo observado à linha de pobreza. Pesquisa e Planejamento Econômico, v. 27, n. 2, p. 313-352, ago. 1997.

SILVA, A. F.; ARAÚJO, J. A.; CAMPELO, G. L.; VASCONCELOS, J. C.; SILVA, J. C. Análise da pobreza multidimensional no Brasil. In: XLII Encontro Nacional de Economia 42, Associação Nacional dos Centros de Pós-graduação em Economia ANPEC -, 2014, Natal, RN. Anais. XLI Encontro Nacional de Economia, Natal, 2014.

STATA - MANUAL. Tetrachoric - Tetrachoric correlations for binary variables. Disponível em: < https://www.stata.com/manuals13/rtetrachoric.pdf>. Acesso em: 03/09/2017.

TRYFOS, P. Chapter 14: Factor Analysis. In Methods for Business Analysis and Forecasting: Text and Case. Versão impressa 2001. Wiley. 1997.

UEBERSAX, J. S. Estimating a latent trait model by factor analysis of tetrachoric correlations. 2000. Disponível em: < http://www.john-uebersax.com/stat/irt.htm>. Acesso em: 24/08/2017.

WESTLUND, H. Form or contents? On the concept of social economy. International Journal of Social Economics, v. 30, n. 11, p. 1192-1206, 2003. 